$\begin{array}{ll} & \text { Etnográfica } \\ \text { etnográfica } & \text { Revista do Centro em Rede de Investigação em }\end{array}$

Antropologia

vol. $24(3) \mid 2020$

Vol. 24 (3)

\title{
Maisons diasporas et maisons locales : mobilités haïtiennes et réseaux transnationaux
}

Diaspora houses and local houses: Haitian mobility and transnational networks

\section{Handerson Joseph}

\section{(2) OpenEdition}

Journals

Édition électronique

URL : https://journals.openedition.org/etnografica/9566

DOI : 10.4000/etnografica. 9566

ISSN : 2182-2891

\section{Éditeur}

Centro em Rede de Investigação em Antropologia

\section{Édition imprimée}

Date de publication : 1 octobre 2020

Pagination : 749-774

ISSN : 0873-6561

\section{Référence électronique}

Handerson Joseph, «Maisons diasporas et maisons locales : mobilités haïtiennes et réseaux

transnationaux », Etnográfica [En ligne], vol. 24 (3) | 2020, mis en ligne le 31 octobre 2020, consulté le

20 janvier 2022. URL : http://journals.openedition.org/etnografica/9566 ; DOI : https://doi.org/

10.4000/etnografica.9566

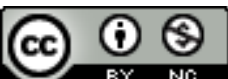

Etnográfica is licensed under a Creative Commons Attribution-NonCommercial 4.0 International License. 


\section{Maisons diasporas et maisons locales : Mobilités haïtiennes et réseaux transnationaux}

\section{Handerson Joseph}

L'article a pour objectif d'analyser les rapports entre maisons et mobilités transnationales dans le monde social haïtien, ainsi que leurs modes de gouvernement. Il s'appuie sur des enquêtes ethnographiques prolongées, menées dans les villes de Fonds-des-Nègres, en Haïti et de Cayenne en Guyane française, permettant ainsi de suivre les trajectoires migratoires des Haïtiens. Est récurrent chez les Haïtiens l'usage du concept « diaspora » pour différencier les maisons : kay dyaspora (maison diaspora), kay lokal (maison locale) et kay nan dyaspora (maison en diaspora). L'article s'intéresse plus particulièrement à deux maisons, une « diaspora » et l'autre « locale » dans le but de montrer comment la construction des maisons et leur ameublement reflètent les liens que les Haïtiens en mobilité transnationale établissent entre eux, les lieux où ils se rendent et leurs maisons d'origine où ils ont parfois un eritaj (héritage). Dans quelle mesure la dynamique des maisons et leur différenciation éclairent-elles le monde social haïtien, notamment les mobilités transnationales de ses acteurs ? Comment, réciproquement, ce monde en mouvement nous renseigne-t-il sur la dynamique des maisons, leurs relations, sur les rapports entre les maisons et les objets qui les peuplent, sur les liens entre ceux qui partent et ceux qui restent sur place? Comment se constitue le gouvernement des maisons ? Il n'y a pas de diaspora sans mobilité des personnes et il n'y a pas de mobilité sans maisons. Les «maisons diasporas » et les « maisons locales » donnent à lire les différentes dimensions de la culture et de la société haïtienne, notamment les hiérarchies socioéconomiques de ce pays.

MOTS-CLÉS : maison diaspora, maison locale, mobilité, Haïti.

Diaspora houses and local houses: Haitian mobility and transnational networks - The objective of the article is to analyze the relationships between homes and transnational mobility in the Haitian social world, as well as their modes of government. It is based on prolonged ethnographic surveys, carried out in the cities of Fonds-des-Nègres, in Haiti, and Cayenne, in French Guiana, thus making it possible to follow the migratory trajectories of Haitians. A recurrent theme among Haitians is the use of the concept « diaspora » to differentiate homes : kay dyaspora (diaspora home), kay lokal (local home) and kay nan dyaspora (home in diaspora). The article focuses more specifically on two homes, one "diaspora" and the other "local" in order to show how the construction of the homes and their furnishings reflect the links that Haitians in transnational mobility establish between them, places they go to in contrast with their original homes where they sometimes have a heritage. To what extent do the dynamics of homes and their differentiation enlighten the Haitian social world, in particular the transnational mobilities of its actors? How, conversely, does this moving world inform us about the dynamics of 
homes, their relationships, the relationships between homes and the objects that populate them, the links between those who leave and those who stay in place? How is home governance constituted? There is no diaspora without mobility and there is no mobility without homes. The "diasporas homes" and the "local home" give an insight into the different dimensions of Haitian culture and society, in particular the socio-economic hierarchies of Haiti.

KEYWORDS : diaspora home, local home, mobility, Haiti.

JOSEPH, Handerson (handersonj_82@yahoo.es) - Programme de post-graduation en Etudes de Frontière, Universidade Federal do Amapá (UNIFAP), Brésil.

EN HAÏTI, LE MOT «DIASPORA » EST UTILISÉ COMME NOM OU ADJECTIF pour qualifier et désigner des personnes, des objets, des monnaies, des maisons et des actions. On le voit dans des expressions telles que «mon rêve est d'être diaspora », «Diaspora, comment vas-tu ? », « ça, c'est des vêtements diasporas », « j'ai de l'argent diaspora », « celle-là est une maison diaspora », « tu agis comme un diaspora », «tu n'es pas n'importe qui, tu es un diaspora », « Diaspora n'est pas Dieu », entre autres. ${ }^{1}$

Le concept de «diaspora» ne désigne pas seulement les compatriotes haïtiens qui résident à l'étranger ou ceux qui reviennent en Haïti pour une courte période avant de repartir. Ils'articule autour de trois verbes : « résider » à l'étranger, « revenir » en Haïti et « retourner » à l'étranger, qui impliquent un ensemble d'actions, de qualités morales et d'états. Quand une "personne diaspora » rêve de « revenir » temporairement en Haïti pour conduire une

l Ailleurs (Joseph 2015b, 2017b et 2019a), j'ai présenté une ethnographie du concept de « diaspora », une pragmatique de ses significations chez les Haïtiens résidant en Haïti et à l'étranger. Les matériaux présentés dans ce texte sont issus de l'enquête ethnographique qui a servi de support à ma thèse de doctorat Diaspora : Les Dynamiques de la Mobilité haïtienne au Brésil, au Suriname et en Guyane Française (en portugais). Dans cette thèse, soutenue en 2015 au sein du Programme de Troisième Cycle en Anthropologie Sociale (PPGAS) du Musée National (MN), l'Université fédérale de Rio de Janeiro (UFRJ), j'analyse les usages sociaux du terme dyaspora et les formes de mobilité propres aux Haïtiens qui sont venus ou passés par le Brésil entre 2010 à 2013, sachant que certains d'entre eux se dirigeaient en fait vers le Suriname et la Guyane française. La première étape du travail de terrain a porté sur la frontière entre le Brésil, la Colombie et le Pérou, notamment, les villes de Tabatinga, et de Manaus (capitale de l'Etat d'Amazonas), entre janvier et février 2012. La deuxième s'est déroulée au Suriname (Paramaribo) et en Guyane française (Cayenne) de mars à mai 2013 et la troisième en Haïti (Fondsdes-Nègres et Pemerle), en juillet de la même année. Une première version de ce texte a été présentée au Colloque International « Gouverner les pratiques économiques », qui a eu lieu à Paris du 26 au 30 septembre 2016, dans le cadre du projet franco-brésilien CAPES-COFECUB « Formes de gouvernement et pratiques économiques ordinaires » (<http://ecogov.weebly.com/ $>$ ). Je remercie le CNPq (Centre National de Développement Scientifique et Technologique brésilien). 
grosse voiture et ainsi montrer le succès de sa trajectoire migratoire, on dit «qu'elle fait des choses de diaspora ». Ce terme assume plusieurs formes et des connotations différentes. Il peut être à la fois un nom, un adjectif et un verbe : « être diaspora ", « avoir des objets diaspora ", «faire des choses diaspora ", construire une «maison diaspora », par exemple.

Le mot de diaspora, qui traditionnellement renvoie à la dispersion du peuple juif par le monde, a considérablement accru sa présence depuis une trentaine d'années, aussi bien dans le discours académique, que dans les médias et même le langage courant, comme dans le cas haïtien. Le mot « diaspora » a pour origine le verbe grec speirein (semer) et le verbe composé diaspeirein (disséminer), qui désigne la dispersion d'une population. Au XVII siècle, il se réfère à la dispersion des choses, des idées et des peuples. Gabriel Sheffer (1986) a été l'un des premiers qui l'a défini avec précision : les diasporas modernes sont des groupes ethniques minoritaires, issus de la migration, qui résident et agissent dans des pays d'accueil tout en maintenant de forts liens affectifs et matériels avec leurs pays d'origine - leurs patries (homelands) ». Au début des années 1990, William Safran (1991) a proposé six critères permettant d'identifier une diaspora : la dispersion d'une population à partir d'un centre vers au moins deux régions périphériques étrangères, le maintien d'une mémoire collective du lieu d'origine, la conscience d'une impossible acceptation par la société d'installation, l'objectif d'un retour dans le lieu d'origine idéalisé, le sentiment d'une obligation collective à défendre le pays d'origine, le maintien à titre individuel ou collectif de liens avec celui-ci. Robin Cohen (1997) a revisité la définition classique de Safran, en y ajoutant quatre autres critères : la migration volontaire, la conscience ethnique sur un temps long, l'apparition d'une créativité nouvelle, une sympathie et une solidarité avec les membres de la même diaspora présents dans d'autres pays. Ces définitions classiques ont été beaucoup critiqués pour leur caractère essentialiste et supposément exhaustif. Des auteurs comme Stuart Hall (1993), Paul Gilroy (1993) et James Clifford (1994) ont notamment développé des approches culturalistes et déconstructivistes, informées par l'expérience de la diaspora noire, qui, contre le modèle de l'Etat-nation, mettent l'accent sur l'hétérogénéité, la diversité et l'hybridité des diasporas.

Dans la suite de ce texte, dyaspora ne renvoie ni au sens original de diaspeirô (disperser) ni à ses définitions analytiques, mais bien à l'interprétation et l'usage qui en est fait dans le monde social haïtien, en Haïti et à l'étranger. L'objectif de l'article est d'analyser les relations entre maisons et mobilités transnationales dans le monde social haïtien. Dans cet univers, la catégorie de maison sert à qualifier des rapports, des traits moraux et des espaces en lien avec ces mobilités. À cet égard, ma démarche s’inscrit dans la lignée de ce que Cortado (2020) a nommé la «nouvelle anthropologie de la maison » : au lieu de considérer la maison comme un lieu fixe remplissant une fonction prédéterminée, 
celle d'héberger le groupe familial par exemple, cette nouvelle anthropologie s'intéresse à la maison en tant qu'elle est pensée et vécue par ses habitants, en tant que « processus complexe d'invention et de réinvention de la famille, de l'amitié, du voisinage et d'initiation à la conjugalité » (Marcelin 1996 : 125). «La maison comme construction physique », disait déjà Louis Herns Marcelin dans sa thèse séminale, " n'est pas séparée des corps qui l'habitent ou qui y transitent, ni des réseaux de personnes qui la modèlent » (Marcelin 1996 : 98). Il se trouve que dans le monde social haïtien comme dans d'autres contextes socioculturels (Marcelin 1999 ; Motta 2014 ; Pina-Cabral 2014 ; Araos 2016, Guedes 2018 ; Cortado 2019 ; Léobal 2019), les maisons sont pensées et vécues en relation les unes avec les autres : elles appartiennent à des « configurations de maisons ». Dans le cas présent, ces configurations se déploient sur des échelles locales, nationales et transnationales.

Les dynamiques sociales et spatiales de la diaspora haïtienne montrent des personnes qui « font partie » de plusieurs maisons à la fois, en Haïti et en diaspora. Parfois même, ils construisent leurs maisons à distance en envoyant de l'argent à leurs proches, qui sont aussi chargés de garder la maison. Au centre de mon analyse se trouve justement la différence entre les maisons construites à partir de ressources locales, les kay lokal ou « maisons locales », et celles bâties grâces aux ressources de la diaspora, les kay dyaspora ou «maisons diasporas ». Comment donc les maisons et leur différenciation éclairent-elles le monde social haïtien, un univers en mobilité ? Comment, réciproquement, le monde en mouvement de la diaspora nous renseigne-t-il sur la dynamique sociale et économique des maisons, les rapports entre maisons et entre les personnes qui partent et celles qui restent sur place ? Quels sont les principes qui organisent ces maisons en Haïti ? D'où viennent les objets qu'elles contiennent ? Comment se gouvernent les «maisons diasporas » et les «maisons locales »?

Je présenterai tout d'abord mon premier terrain en Haïti, où j'ai pu visiter des maisons construites par des Haïtiens qui vivent dans le pays et par d'autres qui sont en diaspora. Je montrerai ainsi comment les «maisons locales » se distinguent des «maisons diasporas ». Je décrirai ensuite la façon dont circulent personnes et objets en prenant appui sur les maisons d'Altamère, de Jinette et de Fanfan. ${ }^{2}$ En plus d'étudier les trajectoires de mes interlocuteurs en Guyane, j'ai observé la façon dont ils s'occupent de leurs maisons, ainsi que les formes de solidarité entre «maisons locales » et «maisons diaspora». En particulier, la construction des maisons et la disposition des objets en leur sein reflètent la façon dont les Haïtiens en mobilité établissent des liens entre les lieux où ils se rendent et leur maison d'origine. C'est là qu'ils reviennent habituellement dans le cadre de leurs obligations familiales, envers les ancêtres et les lwa (divinité, esprit dans le vodou) par exemple, surtout parmi ceux qui pratiquent le 
vodou. Cette contribution montre donc, à partir des réseaux transnationaux et diasporas, que la maison peut être une ressource collective, un lieu à la fois temporaire et permanent, participant à la mobilité des individus, qui n'est pas seulement constitutif des rapports entre les personnes, mais aussi de la circulation des objets, des monnaies, des informations et des liens affectifs.

J'ai pris la décision d'enquêter en Haïti en avril 2013, alors que je me trouvais en Guyane française. Je m'étais rendu compte à cette époque que les primo-arrivants haïtiens des années 1960 et la plupart de ceux qui habitaient ce territoire ultramarin venaient de Fonds-des-Nègres et de Pemerle, deux petits villages au Sud d'Haïti. J'ai donc voulu poursuivre la recherche dans ces deux localités pour comprendre la constitution et le fonctionnement des réseaux transnationaux, en observant la dynamique des maisons et des familles des personnes que j'avais connues à Cayenne ainsi qu'au Brésil.

Quand je suis arrivé en juillet 2013 à Fonds-des-Nègres pour mener un travail de terrain, Jérôme, en plus de m'héberger dans la maison de son cousin qui vivait en France, dont il avait la garde, m'a présenté d'autres personnes et montré leurs maisons. ${ }^{3}$ Le premier jour, en marchant le long de la route de terre qui relie la zone urbaine à la partie rurale de Fonds-des-Nègres, Jérôme me dit : « Ça, c'est une kay dyaspora de quelqu'un qui vit en Guyane française »; « celle-là est une kay dyaspora, son propriétaire vit en France ». J'ai remarqué l'usage du mot dyaspora pour décrire certaines maisons, celles dont les propriétaires vivaient à l'étranger, contrairement à celles dont les propriétaires résidaient à Fonds-des-Nègres et qu'ils appelaient kay (« maison»). Pour les différencier, Jérôme et d'autres utilisaient les expressions kay dyaspora (« maison diaspora ») et kay lokal («maison locale »). Ces maisons se reconnaissaient souvent à leur toiture : alors que celle des «maisons diasporas » est en béton, celle des «maisons locales » est en général fait de tôle (figures l et 2).

La kay dyaspora a son propriétaire qui vit à l'étranger et sa construction est constitutive de cette mobilité internationale. La kay lokal peut aussi avoir des liens avec l'étranger, mais pas de la même manière. En effet, son propriétaire vit sur place, les matériaux de construction et le mobilier de la maison sont souvent achetés localement. Ces maisons peuvent être habitées par des personnes diasporas résidant à l'étranger, mais également par ceux qui sont restés en Haïti. En revanche, les kay nan dyaspora «maisons en diaspora », appartiennent à des Haïtiens dans des pays étrangers, appelés lòt bò dlo (littéralement : « de l'autre côté de l'eau » ou « pays d'outre-mer»), peyi blan (« pays blancs »). ${ }^{4}$

3 Fonds-des-Nègres (en créole, Fondènèg), est une commune d'Haïti, située dans le département des Nippes, dans l'arrondissement de Miragoâne, dans le Sud du pays. Actuellement, sa population totale est de 31.886 habitants, sur une superfície de $92,23 \mathrm{~km}^{2}$.

4 «Peyi blan est une catégorie qui réfère à d'autres pays, mais qui possède plusieurs significations. Elle peut être comprise comme peyi etranje (pays étranger), peyi lòt bò dlo (pays d'outre-mer). Pays blanc désigne les peyi etranje riches, en particulier ceux où on peut gagner un revenu en lajan dyaspora. [continua] 


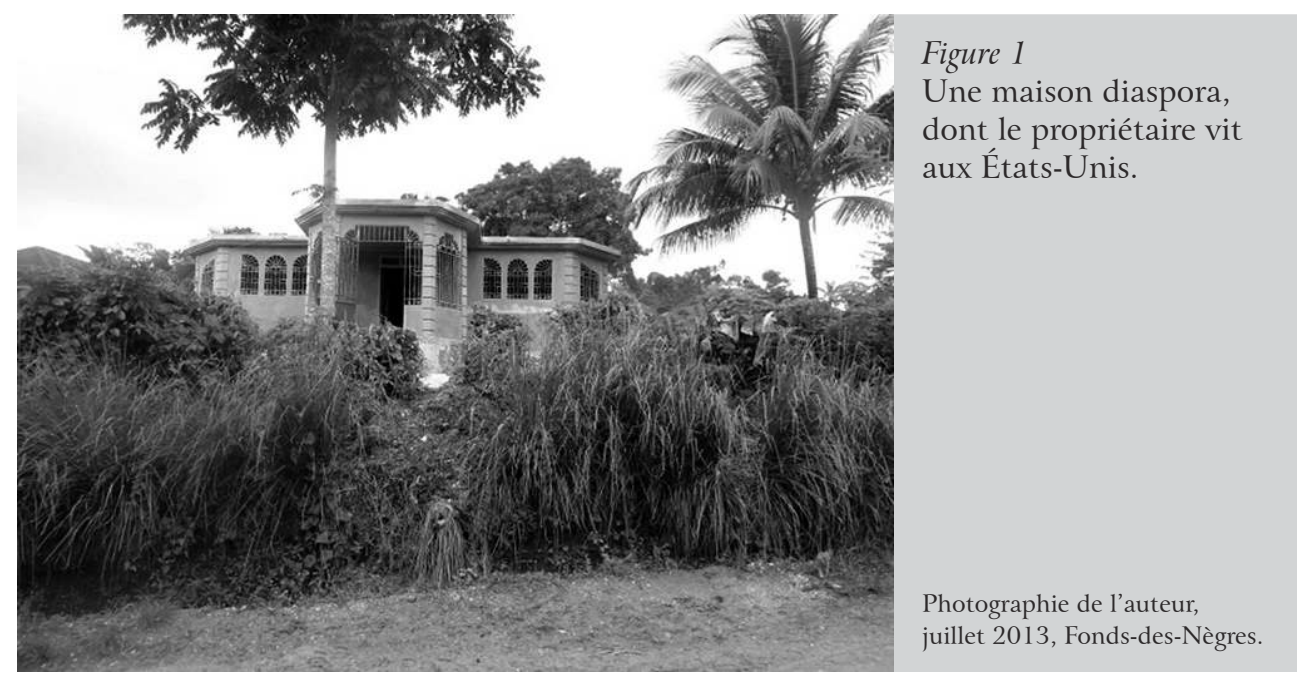

En tant que gardien, Jérôme est le responsable de la maison de son cousin, qu'il surveille depuis sa construction. Il reçoit chaque mois entre 500 et 1000 dollars US de l'étranger pour payer les employés et acheter les matériaux. Il est chargé d'aller à la douane récupérer les matériaux de construction et les appareils électroménagers venus de l'étranger. Il prend des photos ou réalise des vidéos du chantier pour les envoyer à son cousin. Une fois la maison achevée, il y aura sa propre chambre et ayant établi une relation de confiance avec son cousin, celui-ci lui enverra des vêtements et de l'argent pour vivre.

Les «maisons diasporas » que j'ai connues ont mis entre deux et cinq ans à être bâties. Le propriétaire peut se rendre plusieurs fois par an en Haïti pour surveiller le chantier. Celui-ci commence par définir le lieu où construire, acheter le terrain (quand il ne lui revient pas en eritaj, « héritage ») et choisir une personne de confiance pour superviser les travaux. ${ }^{5}$

Les rapports entre le gardien et le propriétaire ne sont pas toujours harmonieux. Parfois, des conflits éclatent, surtout quand le gardien n'obéit pas aux ordres de son patron ou quand il fait un mauvais emploi de l'argent destiné à la construction, en le gaspillant dans des achats inutiles. Dans ces cas,

[continuação] Tous les peyi etranje ne sont pas des peyi blan. Par exemple, la République dominicaine, le Panama, l'Equateur et le Pérou sont des peyi etranje, mais ne sont pas considérés comme des peyi blan. En règle générale, les peyi blan sont des pays industrialisés, « développés » comme les États-Unis, le Canada, la France ou l'Angleterre. Dans le cas de la Guyane, mes interlocuteurs qui résidaient là-bas ne semblaient pas toujours la considérer comme un peyi blan à cause du climat et parce qu'on n'y voyait pas de grands bâtiments modernes. En revanche, quand ils parlaient de la Guyane en tant que département d'outre-mer, ils la qualifiaient de peyi blan, car elle était dirigée par le gouvernement français (Joseph 2017a : 196).

5 Le terme «eritaj» désigne des biens immobiliers, une maison, un lakou, etc. 


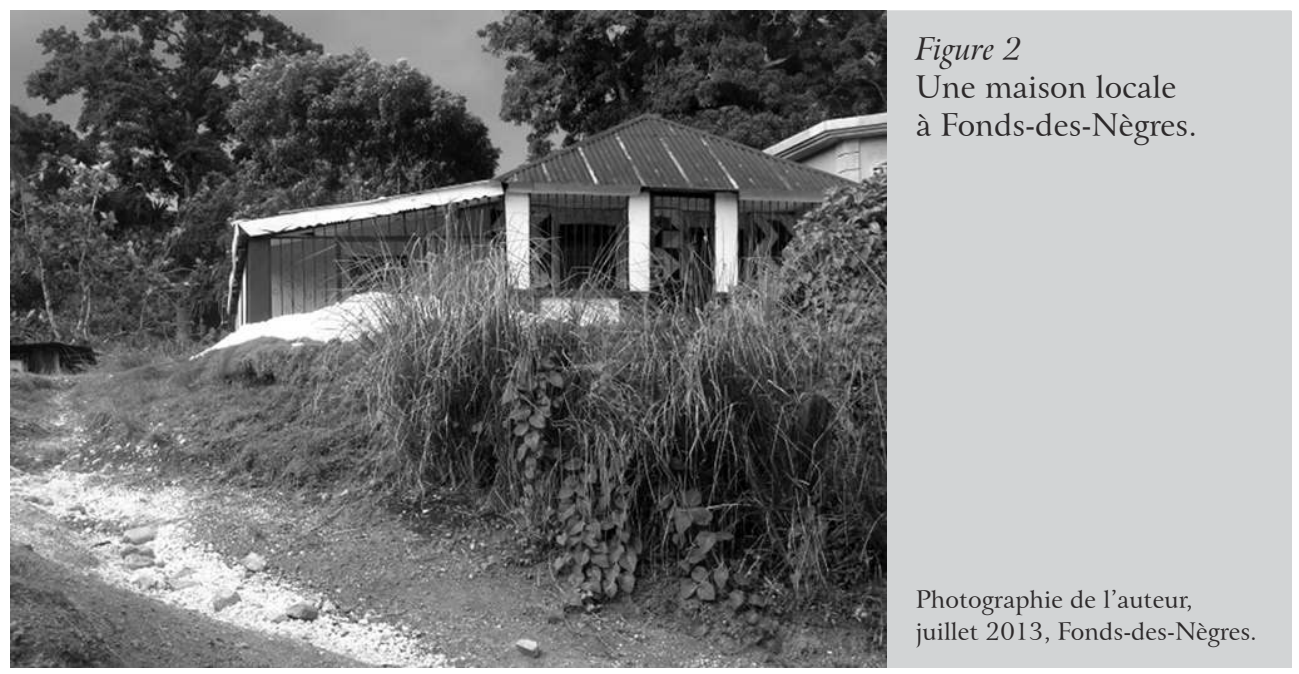

le propriétaire peut rompre avec le gardien et choisir une autre personne de confiance, comme un membre de la famille. Cependant, choisir quelqu'un de la famille peut alimenter la jalousie parmi les autres membres. Quand la maison n'a qu'un propriétaire, c'est lui qui choisit le gardien, mais il peut demander ou non des conseils aux autres. Dans le cas contraire, le choix du gardien est décidé entre les différents propriétaires, qui mobilisent leurs réseaux sociaux et familiaux respectifs. La construction de la maison participe ainsi à la création et recréation de ces réseaux.

Dans le cas d'une kay lokal, il n'y a pas forcément de gardien, puisque le propriétaire vit souvent sur place, sauf quand il s'agit d'une maison construite par un habitant d'une autre ville. La question du « gouvernement de la maison » (Foucault 1984; L'Estoile 2014), de la façon dont est distribué le pouvoir dans et entre les maisons et de ce sur quoi il s'applique, ne se pose donc pas dans les mêmes termes entre une «maison diaspora » et une «maison locale ». Dans le cas de la «maison diaspora », on compte trois niveaux : les relations stratégiques entre les individus, notamment entre le gardien et le propriétaire mais aussi entre ceux qui vivent dans des maisons appartenant au même réseau ; les techniques de construction et l'organisation des « maisons diasporas » et « locales»; enfin, l'occupation des espaces habités et la disposition des objets dans ces maisons. Ces différents niveaux seront mis en évidence dans l'analyse des maisons d'Altamère, de Jinette et de Fanfan.

\section{JINETTE ET LES «MAISONS DIASPORAS »}

À Fonds-des-Nègres, Jérôme m’a présenté à Jinette, née le 3 novembre 1956 à Anse-à-Veau. Elle est mère de deux enfants, Karol, 25 ans, qui vit avec elle à 


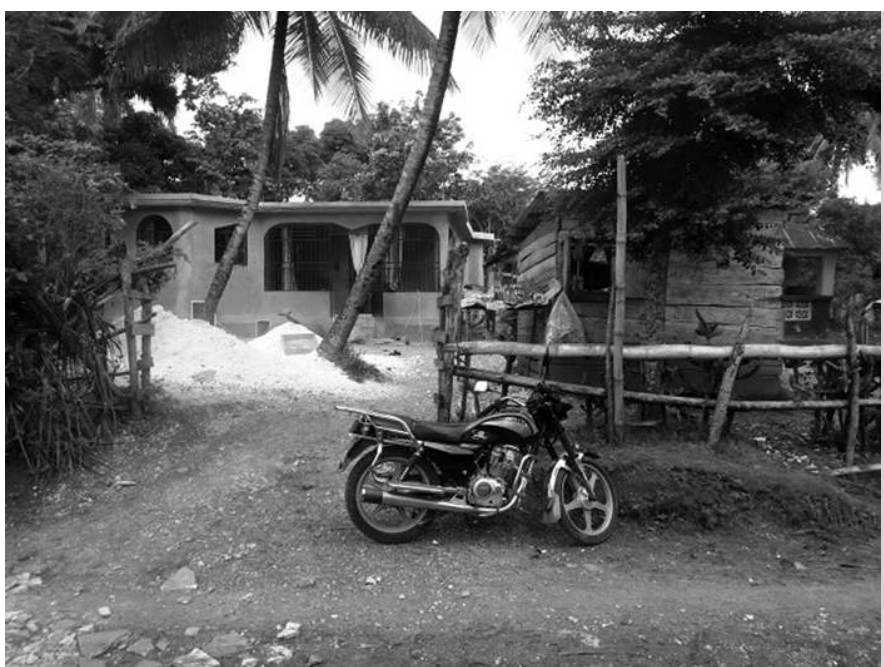

Figure 3

À côté de la maison de Jinette, peinte en jaune, se trouve la « maison diaspora » de sa soeur Altamère ; devantle «petit commerce» (ti biznis) en bois.

Photographie de l'auteur, juillet 2013, Fonds-des-Nègres.

Fonds-des-Nègres et Kendi, 30 ans, parti au Brésil en 2011 et qui vit actuellement à Cayenne (Joseph 2020). À l'époque de l'enquête, Jinette vivait dans la maison avec quatre personnes : sa fille, son petit-fils de douze ans (fils de Kendi), un fils adoptif de treize ans (fils de sa sœur) et une fille adoptive de quatorze ans.

Depuis 1991, la mère de Jinette vit à Paris et son père dans un village près de Fonds-des-Nègres. Elle a deux sœurs aînées, respectivement du côté de sa mère et du côté de son père. L'une vit à Cayenne et l'autre à Paris. Jinette a aussi vécu en Guyane dans les années 1990. Ses deux sœurs lui envoient de l'argent tous les deux ou trois mois pour acheter à manger. Altamère, vivant à Paris, envoie généralement des vêtements pour les enfants et aussi à Jinette pour qu'elles les revende dans son «petit commerce» (ti biznis). Selon Jinette, lajan kay la ( « l'argent de la maison ») ${ }^{6}$ provient d'abord de ti biznis (elle vend riz, haricots, sucre, sel, épices, et autres en petite quantité). C'est lui qui permet notamment de payer chaque mois l'école des enfants. La façon dont est géré « l'argent de la maison » met en évidence la distribution du pouvoir dans la maison et ce sur quoi il s'exerce. Jinette est ainsi la gardienne de sa maison et de celle d'Altamère. Pour employer une expression haïtienne, elle est la poto mitan de la maison («pilier de la maison»). C'est donc elle qui reçoit l'argent de l'étranger pour la construction de la maison de sa sœur. D'un autre côté, son commerce, ses animaux et ses cultures maraîchères lui offrent des ressources stratégiques : si nécessaire, ils seront vendus pour payer les dépenses liées à la maladie et les frais de scolarité.

6 En un sens très proche de celui mis en évidence par Motta (2014) dans son ethnographie des favelas de Rio de Janeiro. 


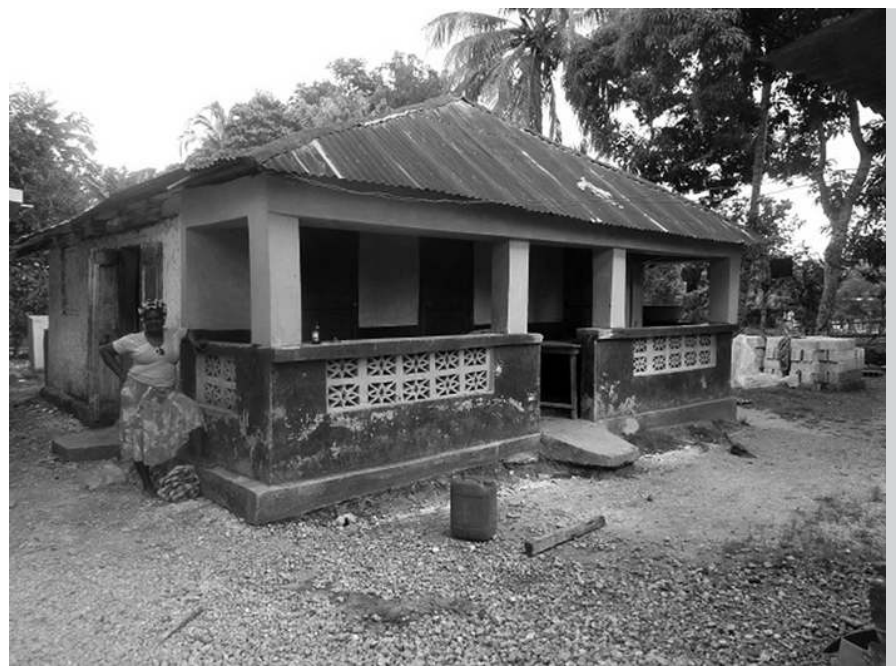

Figure 4

Jinette et sa maison.

Photographie de l'auteur, juillet 2013, Fonds-des-Nègres.

La maison de Jinette (figure 4) n'est plus habitée, elle sert à ranger les outils, stocker les récoltes du jardin ainsi que la nourriture des animaux. Sa sœur qui vit à Cayenne l'a aidée financièrement dans la construction. Jinette raconte avec humour que pour construire sa propre «maison locale », le ciment a dû être transporté sur des ânes, les voitures n'ayant alors pas accès au site. Dans le cas de la maison d'Altamère (figure 5), les matériaux ont été acheminés par camions. Depuis quelques années, Jinette vit dans la «maison diaspora » de sa soeur Altamère.

La mère de Jinette a reçu un lakou comme eritaj de ses parents. ${ }^{7}$ Comme elle a déménagé à Paris et que ses deux autres filles vivent l'une à Paris, l'autre en Guyane, seule Jinette continue en Haïti. Si ce lakou a été transmis en eritaj à Jinette, Altamère et Arnette, il est donc sous la garde de Jinette. Dans ce même lakou, en 2009, Altamère, qui maintenant vit à Paris, a commencé la construction d'une «maison diaspora » à côté de la «maison locale » de Jinette. La «maison locale » de Jinette et la « maison diaspora » de sa sœur se situent, de fait, dans le même lakou.

Après le tremblement de terre de janvier2010, Altamère, qui vivait à Paris, s'est rendue en Haïti en juin. Sa mère, Carmen,quin'était pas rentrée au pays depuis vingt ans l'accompagnait. ${ }^{8}$ Le séjour de Carmen et d'Altamère en Haïti

7 Selon Paul Moral (1961 [1978] : 171), «lakou fait référence à l'agglomération de petites maisons autour d'un terrain au milieu du jardin ». Les travaux sur le vodou (Métraux 1958) et l'univers rural haïtien (Moral 1961 [1978] ; Barthélemy 1990, entre autres) décrivent le lakou comme une forme d'organisation sociale et économique, un ensemble de plusieurs maisons appartenant à une seule famille et de sites dédiés aux rituels vodous et au culte des ancêtres.

8 Elle était venue à Paris dans le cadre du regroupement familial, grâce à une nièce légalement tenue pour sa fille (elle l'avait enregistrée sur son acte de naissance). 


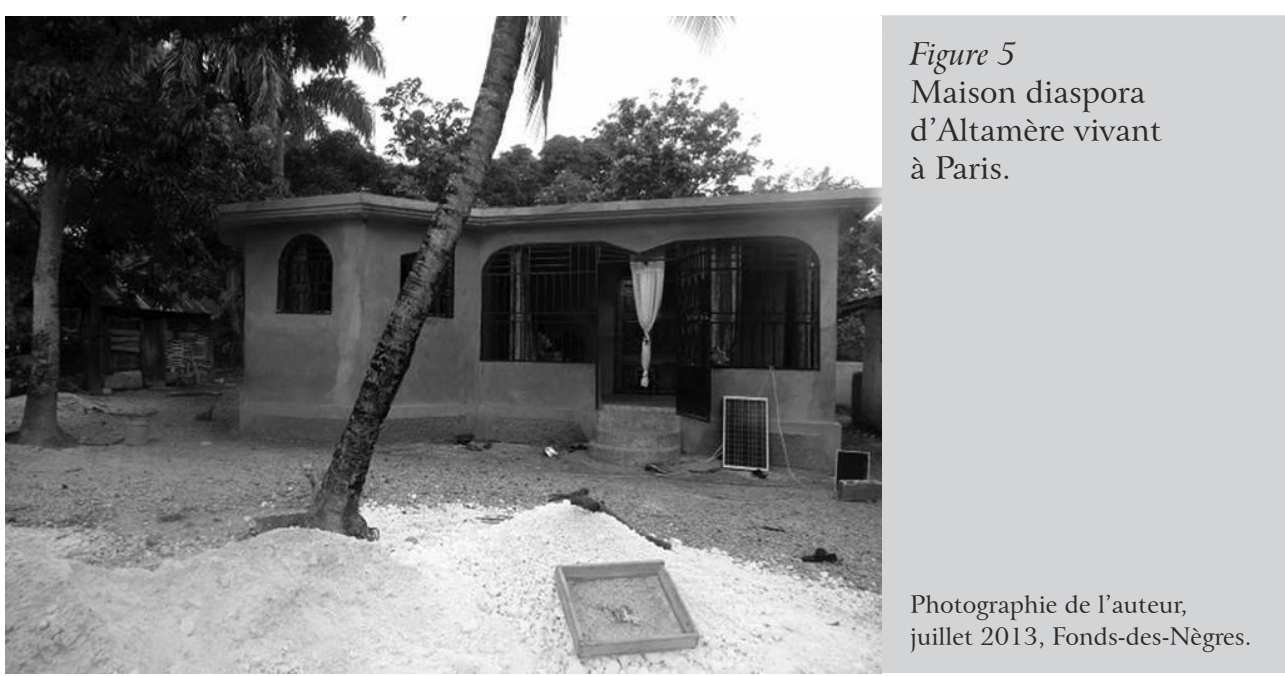

a donc signifié les retrouvailles entre la mère (Carmen) et la fille (Jinette) d'une part, et entre l'arrière-grand-mère (Carmen) et l'arrière-petit fils Standley, fils de Kendi, d'autre part. Il a permis à Altamère de rendre visite à sa famille et aussi de superviser sa «maison diaspora », notamment de couler la chape de béton qui sert de toiture et de construire le plancher de la maison.

Pour ceux qui bâtissent une "maison diaspora », gouverner la maison c'est également la superviser à chaque séjour en Haïti, afin de vérifier si ceux restés sur place ont bien respecté les consignes reçues de l'étranger, si le projet avance bien ou tout simplement l'état de la maison. Ces retours servent aussi à régler les problèmes bureaucratiques et juridiques liés à la maison. Le propriétaire en profite pour fê règleman kay la (littéralement : «faire règlement de la maison ») et le gardien pour bay règleman kay la (« donner règlement de la maison »), notamment en ce qui concerne le budget de la maison. «Faire» et « donner règlement » de la maison sont deux verbes qui renvoient à deux façons différentes d'exercer le pouvoir au sein de la «maison diaspora».

Ce séjour a révélé le succès de l'entreprise migratoire à la famille et aux voisins. Avant de venir en Haïti, Altamère avait envoyé par conteneur des « objets diasporas » : deux cuisinières, des appareils électroménagers, un panneau solaire et un delco (générateur d'électricité). ${ }^{9}$ À son arrivée à

9 À Fonds-des-Nègres comme dans une grande partie du pays, à l'exception de quelques endroits à Port-au-Prince et dans les grandes villes, l'accès à l'électricité n'est pas assuré en continu. Environ trois à huit heures par jour, l'entreprise Électricité d'Haïti (EDH) répartit l'électricité entre différentes localités. Aussi, la zone urbaine de Fonds-des-Nègres se retrouve parfois sans courant. Ceux qui ont les moyens achètent donc des générateurs d'électricité, comme un delco, ou des panneaux solaires pour garantir leur approvisionnement. Ceux qui n'en ont pas les moyens recourent à des méthodes traditionnelles telles que la lampe tèt gridap - une lampe à kérosène - pour au moins éclairer la maison. 
Fonds-des-Nègres, ces équipements se trouvaient déjà dans la maison. Lorsque des Haïtiens reviennent à Fonds-des-Nègres, ces « personnes diaspora » font eux-mêmes transporter par conteneur une grande caisse (droum) contenant des vêtements, des appareils électroménagers et des aliments (pâtes, huile, riz, etc).

Tibiznis

Devant les deux maisons

kay local de Jinette

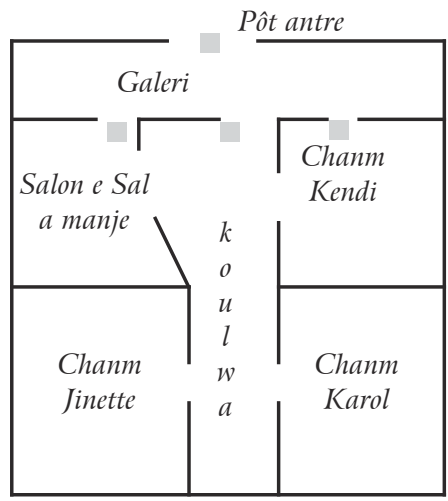

Basin

Pour capter l'eau de la maison kay dyaspora d'Altamère

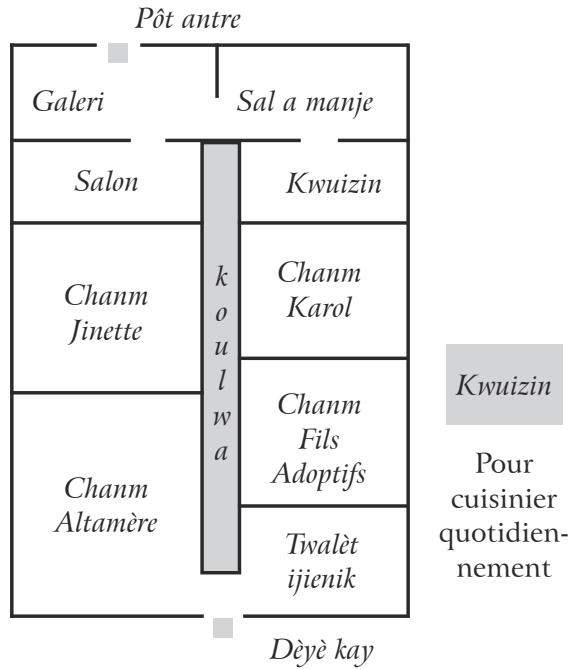

\section{Latrin}

Latrine kay lokal

Dans cet espace les habitants plantent de l'igname, des haricots, du maïs, des pommes de terre, du manioc et du café et les animaux circulent.

Figure 6-Lakou de Jinette et les maisons. 


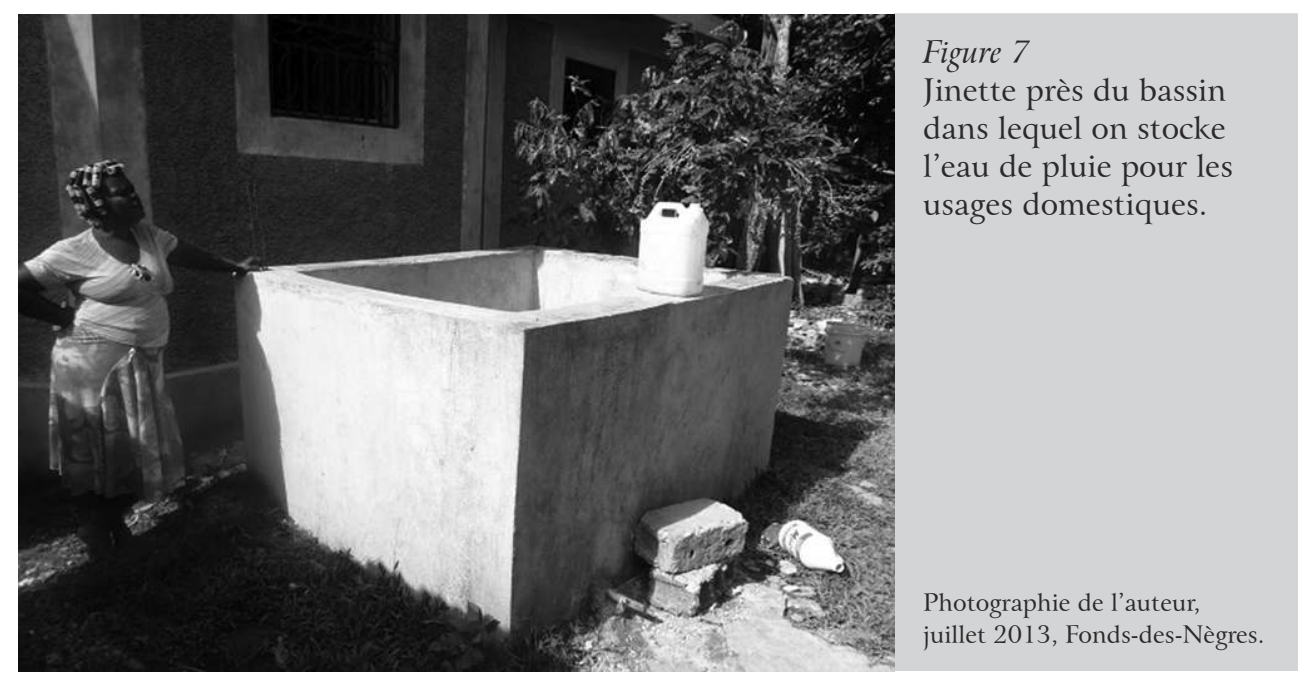

Jinette et son petit-fils Standle y occupent la même chambre, sa fille Karol une autre et les deux autres filles adoptées encore une autre. Celles-ci sont nées à Fonds-des-Nègres et vivent avec Jinette depuis la naissance. La dernière chambre garde les effets d'Altamère. Personne ne devrait y rentrer, seule Jinette en possédant la clé. Selon Jinette, si quelqu'un rentrait dans cette pièce sans son autorisation, il violerait automatiquement sa confiance et celle d'Altamère. La clé est un moyen de contrôler la circulation dans la maison et donc une façon de gouverner les personnes, les objets et l'espace de la maison. Seules Jinette et Altamère déterminent qui peut entrer dans la maison, notamment dans la chambre d'Altamère.

À gauche de la «maison diaspora », il y a un bassin en béton qui sert à capter l'eau de pluie pour les « besoins du ménage », comme faire la cuisine, laver et nettoyer les ustensiles (figure 7). Ce bassin est appelé « l'eau de la maison » (dlo kay la), aussi utilisée dans la construction. ${ }^{10}$

Il y a une cuisine à l'extérieur, d'usage quotidien, l'autre à l'intérieur étant réservée aux visites des «personnes diasporas », venues de l'étranger ou pour des fêtes. Dans une chambre de la «maison diaspora» que fait construire Altamère, il y avait six grandes valises : celles-ci sont importantes pour caractériser la «maison diaspora », en exprimant concrètement la mobilité des habitants.

10 Il n'y a pas de réseau d'eau à Fonds-des-Nègres. Les alternatives sont la construction de bassins, qui captent l'eau de pluie, ou celle de $p i$ (puits), creusés à quelques mètres de profondeur pour puiser l'eau du sous-sol. Dans les zones urbaines de Fonds-des-Nègres il y a les pomp dlo (pompes à eau) installées par la Mairie (figure 8). Ces pompes ne sont pas toujours construites par l'Etat et les administrations publiques : certaines sont l'initiative d'organisations non-gouvernementales opérant dans la région ou sont financées par les membres de la diaspora qui ont leurs maisons sur le site. 


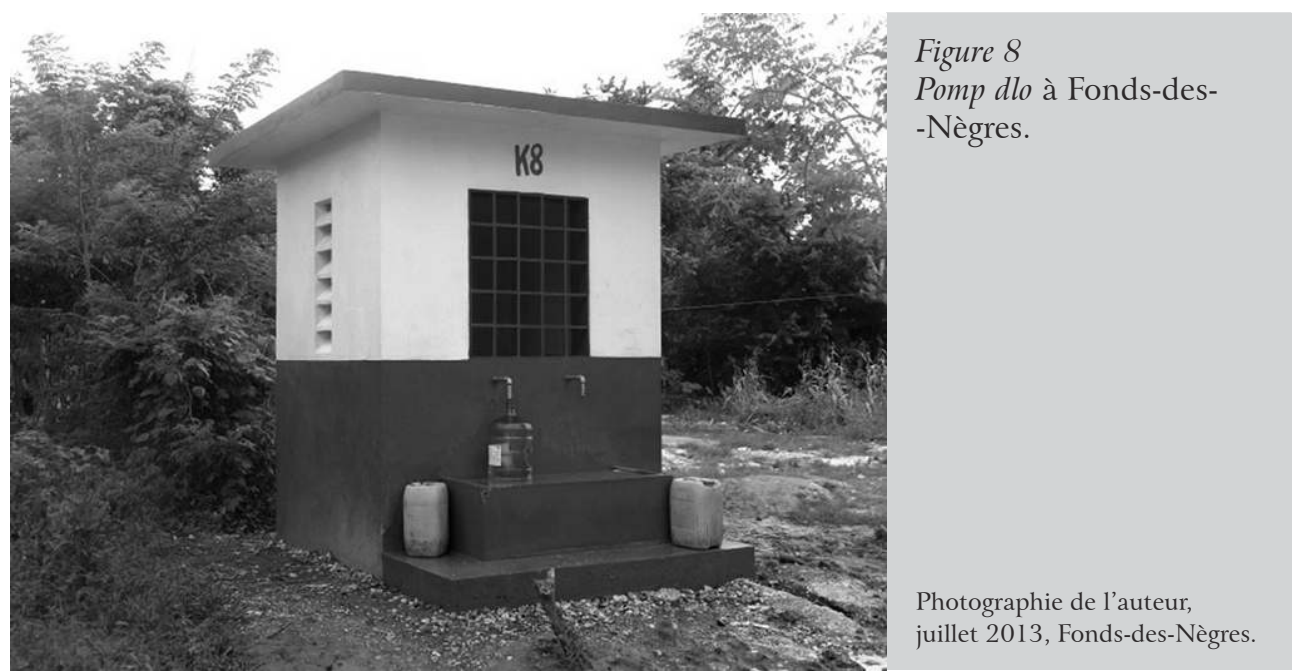

FANFAN ET LA « MAISON LOCALE »

En juillet 2013, Jérôme m'a emmené chez Fanfan pour faire sa connaissance. À l'époque, Fanfan vivait avec ses deux enfants ; son épouse, Lucette, habitait au Brésil depuis 2011. Le même jour, j’ai également rencontré Gislène, qui elle aussi venait rendre visite à Fanfan. Gislène est la femme de Luck, frère de Lucette, lui aussi au Brésil depuis 2011.

À cette époque, Fanfan et Gislène détenaient déjà un visa brésilien, obtenu dans le cadre du regroupement familial. Fanfan était en train d'organiser son départ pour le Brésil et de réunir les fonds pour acheter son billet d'avion et ceux de ses deux enfants, qui à l'époque coûtaient 1400 dollars US chacun. En outre, il préparait trois valises, la sienne et celles de ses deux fils, pour retrouver Lucette à Manaus, au nord du Brésil. Pour partir, en plus des fonds qu'ils ont eux-mêmes réuni, Luck et Lucette ont reçu le soutien de parents vivant en Guyane, à hauteur de 4500 dollars US chacun. Enfin, ils ont emprunté - en créole, pran ponya - auprès d'un usurier connu à Fonds-des-Nègres. À l'époque où Lucette et Luck ont trouvé du travail au Brésil, ils payaient déjà des intérêts.

Quelques mois après s'être établis à Manaus, Lucette et Luck ont commencé à envoyer de l'argent à Fanfan et Gislene, restés en Haïti. Lucette envoyait tous les deux mois entre 100 et 150 dollars à son mari Fanfan pour acheter à manger. Quand j'ai interrogé Gislène sur le montant reçu de son mari Luck, elle a ri quelques secondes, puis a déclaré : « 80 à 100 dollars tous les deux mois ». Selon Gislène, ceux qui habitent aux États-Unis, en France et au Canada, c'est-à-dire dans les " pays blancs », pourraient envoyer plus d'argent que ceux qui sont restés en Haïti, puisqu'ils ont de meilleurs emplois 


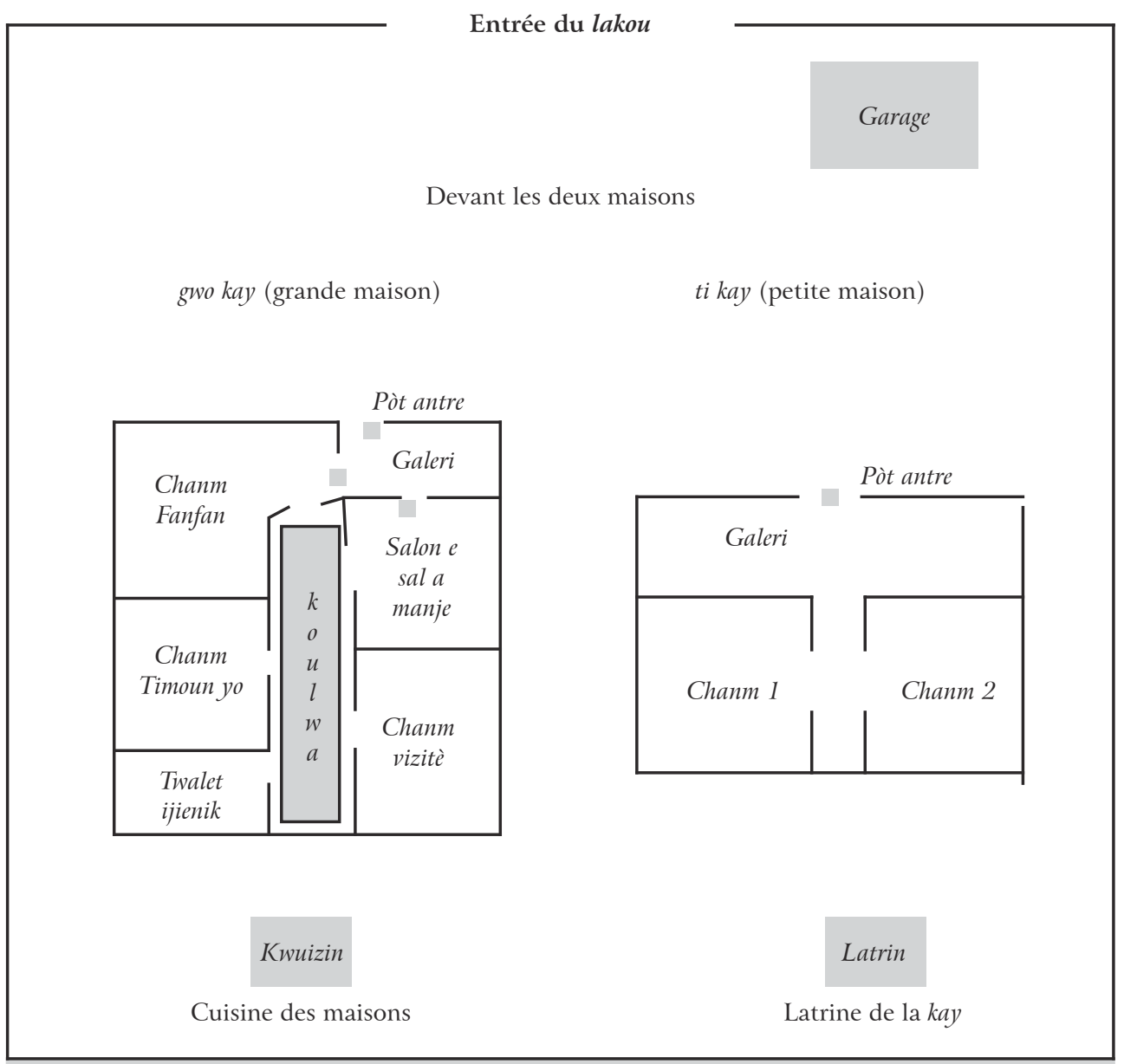

Figure 9 - Lakou et maison de Fanfan.

et gagnent des salaires élevés. En outre, ils sont payés en lajan dyaspora et kòb dyaspora, notamment en dollars américains et en euros (Joseph 2015a, 2015b, 2019b), devises à la valeur symbolique, sociale et marchande largement supérieure.

Fanfan a expliqué la mobilité de sa famille de la manière suivante : «En règle générale, à cause de la situation socioéconomique en Haïti, la personne est obligée de se déplacer et, plus encore quand elle n'a pas de ressource financière pour survivre, lorsqu'une occasion de partir se présente, elle s'en va ». Sa femme Lucette est ainsi partie pour trouver du travail. En même temps, ce départ a causé une certaine frustration : « Ça n’a pas été facile, le fait qu'elle parte [Lucette] et nous laisse ici, c'est une séparation, mais elle en avait davantage besoin que moi. Même si je ne gagne pas beaucoup, j'ai mon travail ici ». Lucette travaillait à Manaus comme agent d'entretien dans un 


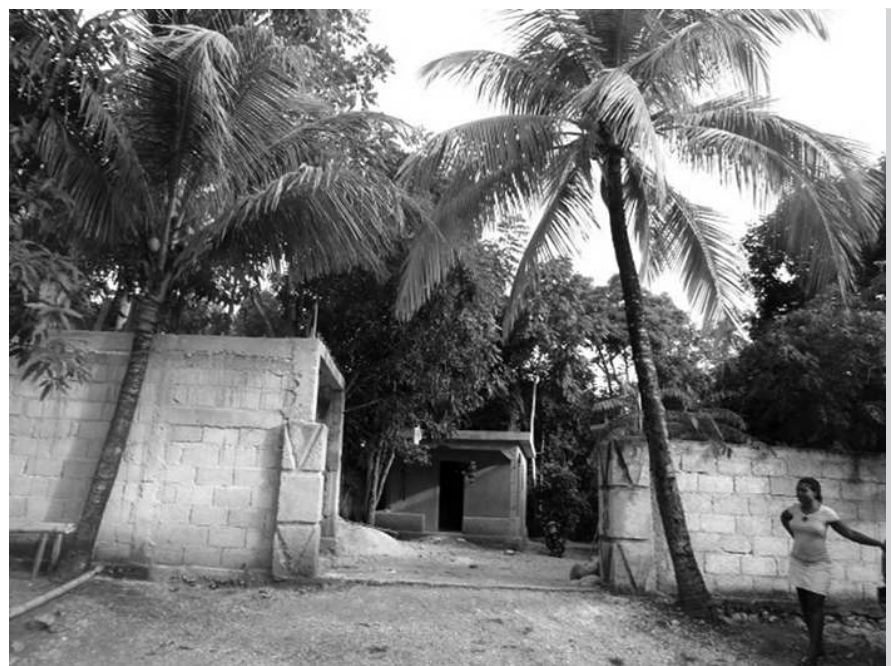

Figure 10

Gislène en face de la maison de Fanfan.

Photographie de l'auteur, juillet 2013, Fonds-des-Nègres.

hôpital. Fanfan poursuit : «Je ne suis pas tout à fait désolé, parce que maintenant les enfants auront l'opportunité de faire des études là-bas [au Brésil], après ils peuvent revenir pour servir leur pays ». Pour Fanfan, faire leurs études à l'étranger confère à ses enfants une valeur morale et sociale. Au retour en Haïti, ils auraient ainsi accès à de meilleures opportunités.

Il y a dix-neuf ans, Fanfan et Lucette ont bâti une maison à Fonds-desNègres. Pour réaliser ce rêve, ils ont pris sur le salaire de Fanfan, le peu qui restait du commerce de Lucette et ont reçu une aide financière de leurs amis. Son lakou était entouré d'un mur élevé qui abritait la maison principale, appellé gwo kay ( "grande maison ») et, à côté, une autre plus petite, ti kay («petite maison ») en chantier depuis 2011 , avant le départ de Lucette (figure 10). Fanfan et Lucette étaient les seuls résidents de ce lakou. En face de ces deux maisons, il y avait un garage, dont la construction a commencé en 2012 . Toutefois, ils n'avaient pas de voiture à l'époque. Selon Fanfan, « nous avons fait le garage parce que nous voulons avoir une voiture un jour ». La petite maison dispose de deux chambres et d'une galerie. Parfois, Lucette envoyait de l'argent depuis le Brésil pour continuer le chantier. C'est avec cet argent que Fanfan a construit la galerie de la petite maison.

Avec ce qu'il restait de sable, ciment et parpaing, Fanfan a pris l'initiative de bâtir une petite maison à gauche de la maison principale, ce qui n'était pas dans les plans du couple. La « grande maison » possède une galerie, un salon avec salle à manger, trois chambres et une toilette et salle de bains en construction. Pour le moment, ils se servent des latrines situées au fond du lakou et de la cuisine pour préparer à manger. Pendant l'absence de sa femme, Fanfan dort dans la chambre à coucher du couple, les deux enfants dans une autre, la dernière est réservée aux invités. 


\section{LA RELATION ENTRE MAISONS ET ESPACES TRANSNATIONAUX}

À la lumière des trajectoires de Jinette et de Fanfan, et des données du travail de terrain en Guyane, on examinera ici la relation entre les «maisons diasporas » et les «maisons locales », leurs hiérarchies et le gouvernement des maisons. La construction des maisons et les objets qui s'y trouvent reflètent la façon dont les émigrés tissent des liens entre Haïti, les réseaux transnationaux et les diasporas.

Au cours des années 1990, Gérard Barthélemy a justement exposé ces liens dans ses travaux sur les maisons construites par les émigrés haïtiens en milieu rural :

« Ce rapport permanent à l'extérieur qui caractérise la plupart des sociétés caribéennes et notamment celle-ci, est, nous l'avons vu, un élément inhérent au système de l'autorégulation. Il est en même temps, en raison du flux permanent d'échanges avec l'extérieur qu'il suscite, une source de nombreuses transformations. Ainsi en milieu rural, l'habitat tend actuellement à se stabiliser et à se 'durcir' dans des structures bétonnées en raison des exigences propres aux constructions financées par les émigrés, pour eux-mêmes ou pour leur famille» (Barthélemy 1990 : 173-174).

Lauteur associe la construction des maisons en Haïti au processus migratoire, soulignant quelques différences structurelles entre les maisons construites par les migrants lors de leur retour en Haïti et les autres. À une autre échelle d'analyse, des travaux ont montré le rôle des objets réutilisés ou envoyés dans les pays du Tiers-monde dans le contexte contemporain des communautés mondialisées (Peraldi 1999, entre autres). D’autres ont porté sur la culture matérielle des mondes domestiques, l'archéologie de la vie culturelle et les processus par lesquels les individus s'approprient des objets, en donnant un sens aux gens et aux mondes dans lesquels ils opèrent (Pertierra 2010).

Il est important de rappeler qu'en Haïti, bon nombre de ceux qui partent à l'étranger construisent des maisons à louer et/ou pour se loger à leur retour. Habituellement, la maison est meublée avec des « objets diasporas » exportés depuis le pays de résidence du propriétaire, comme des lits, armoires, réfrigérateurs, téléviseurs, lecteurs DVD, climatiseurs, ventilateurs, cuisinières, canapés, vaisselle, couverts, etc. Si le mobilier d'une «maison locale», appelé «meubles locaux », était autrefois en fer, de nos jours, il est majoritairement en bois. Pour construire certaines "maisons diasporas », les gens se servent de matériaux fournis par la diaspora, comme on a observé dans le cas d'Altamère qui réside en France. La céramique, les portes, les fenêtres, les planchers et les lustres sont transportés par bateau, du pays d'installation vers Haïti, dans des conteneurs. Afin de brancher leurs appareils ramenés de l'étranger, certains adaptent leur 
réseau électrique pour le mettre aux normes étrangères (par exemple, 220 volts pour ceux qui viennent de la France, alors que la norme est 110 en Haïti) ou apportent leur générateur, appelé « delco », et des panneaux solaires.

Dans la majorité des maisons en Haïti, ce sont les femmes qui préparent à manger et distribuent les repas dans les réseaux de maisons. Il y a une relation étroite entre le genre/sexe, l'âge et la distribution de la nourriture. Géneralement, quand les femmes vivent en couple, elles servent l'époux d'abord, puis les enfants de sexe masculin selon leur âge, du plus âgé au plus jeune, et enfin les femmes, en commençant par la plus âgée. ${ }^{11}$ Cette hiérarchie créé un rapport inégalitaire entre les hommes et les femmes dans le gouvernement de la maison : même si les femmes contrôlent la cuisine, ce sont les hommes qui occupent le sommet de la hiérarchie quand il s'agit de distribuer la nourriture, établissant ainsi une différence entre gouverner la maison, gouverner la cuisine et gouverner la nourriture. L'emprise feminine sur la cuisine ne veut pas dire que les femmes dominent totalement la maison, même si parfois elles en sont le pilier, comme on a pu l'observer dans le cas de Jinette.

Larchitecture, la taille des pièces, le style de la salle de bain, les modes d'organisation et les façons de gouverner l'espace habité diffèrent entre « maison diaspora » et «maison locale ». La cuisine des «maisons diasporas » se situe à l'intérieur de la maison principale, parfois séparée de la salle à manger par un simple comptoir en béton ou en bois (cuisine américaine). Dans les «maisons locales », la cuisine est séparée du bâtiment principal. Faite de bois, paille, terre ou béton, elle se trouve à quelques mètres à côté ou derrière la maison. ${ }^{12}$ Parfois elle sert aussi de réserve pour stocker le recho (réchaud, type de cuisinière en aluminium très populaire en Haïti), le charbon, les fruits et les légumes. Alors que dans les «maisons diasporas » on se sert d'une cuisinière, dans la majorité des « maisons locales », c'est le charbon qu'on met dans le recho. La cuisine, dans les deux types de maison, est le lieu par excellence de la sociabilité entre proches, amis et voisins.

Dans une "maison diaspora », la salle de bains se divise entre l'espace de la douche, le lavabo et les toilettes. La salle de bain est appelée twalèt ijienik («toilette hygiénique », figure 11). Comme il n’y pas d'égouts pour canaliser les matières fécales, les toilettes d'une « maison diaspora » sont reliées à des fosses septiques. Dans la «maison locale », loin à l'arrière du bâtiment principal, il y a des latrines (figure 12). Comme il n'y a pas de douche, les gens

11 Cela ne veut pas dire que toutes les maisons haïtiennes suivent le même modèle : il y en a qui ne suivent pas cette hiérarchie des sexes et des âges. Cela dépend de la composition familiale, du niveau d'étude et de qui sont les responsables de la maison.

12 Il y a un aspect pratique au fait que la cuisine se situe derrière le bâtiment principal : quand il pleut, le sol en terre battue se transforme en boue et il devient plus difficile de circuler entre le bâtiment principal et la cuisine sans salir la maison. 


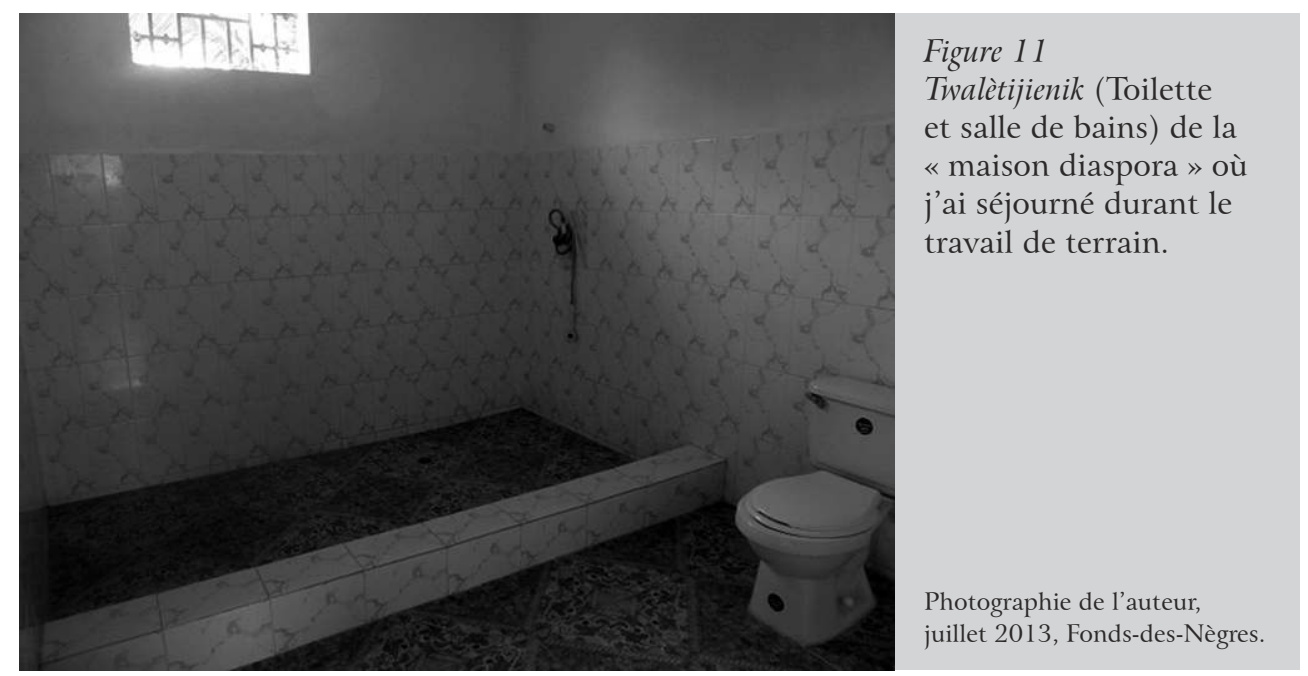

font souvent leur toilette à l'air libre. Les fosses septiques se trouvent généralement à quelques mètres ( 15 à $20 \mathrm{~m}$ ) de profondeur avec une structure en bois par-dessus pour pouvoir mettre ses pieds ou s'asseoir. Quand les latrines sont collées à la salle de bain, une cloison en bois, en béton ou juste un rideau servent à les séparer. Pour prendre une douche, on se sert de seaux (bokit) et d'un bol en plastique pour verser l'eau. Ces éléments annoncent aussi le statut social du propriétaire de la maison et ainsi le pouvoir économique de la famille qui y habite.

Il y a donc une hiérarchie des styles architecturaux en Haïti, la kwuizin avec gazinières s'opposant à la kwuizin avec recho, les twalèt ijienik aux latrin, l'électricité à la lampe tèt gridap. Cette hiérarchie s'étend à l'idée même de maison. En effet, les «maisons diasporas » sont souvent plus riches que la majorité des «maisons locales », même s'il y a des exceptions. En ce qui concerne la finition, la «maison diaspora » est habituellement peinte, mais pas toujours. Les autres sont le plus souvent dépourvues de revêtement. Les couleurs des «maisons diasporas » varient en accord avec le goût du propriétaire et l'esthétique du lieu où il réside.

En général, la classe sociale à laquelle appartiennent les propriétaires, leur capital culturel acquis en Haïti et à l'étranger, la durée plus ou moins longue de leur résidence à l'extérieure, leur lieu d'émigration, le nombre de « personnes diasporas » impliquées dans la construction et la présence de la famille sur place influent significativement sur le résultat des travaux. Il y a plusieurs types de «maisons diasporas », en accord avec les différentes caractéristiques de leur propriétaire.

La diversité des trajectoires résidentielles fait que riches et pauvres peuvent se côtoyer au sein d'un même réseau domestique et familial. C'est pourquoi on 


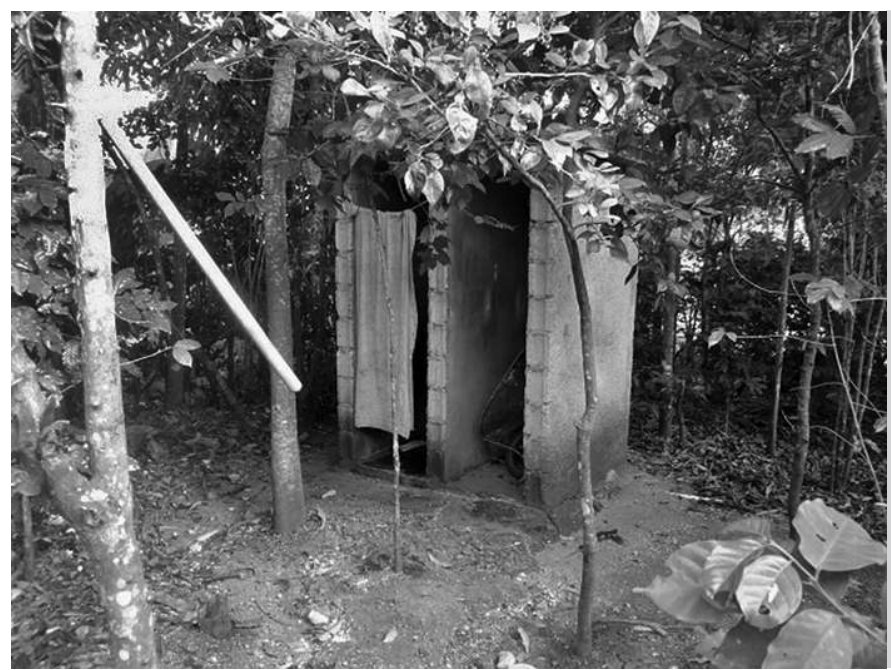

Figure 12

Twalèt (salle de bains) et latrin (latrines) l'une à côté de l'autre dans une « maison locale.

Photographie de l'auteur, juillet 2013, Fonds-des-Nègres.

dit souvent en Haïti qu'il est « impossible que quelqu'un n'a jamais vu la pauvreté », dans la mesure où riches et pauvres sont fréquemment amenés à vivre ensemble. On peut être riche en Haïti, mener une vie bourgeoise, mais dans la même rue ou le même quartier habitent des gens plus pauvres, qui peuvent être membres de la famille, voisins et amis.

La disposition des espaces, la construction de la maison, les relations avec les autres maisons expriment et produisent des hiérarchies entre individus appartenant à la même famille. Comme une «maison diaspora » peut appartenir au même réseau qu'une « maison locale », gouverner la maison, en certains cas, signifie gouverner un réseau hétérogène de maisons, qui renvoie à des catégories strictement hiérarchisées de maisons et de personnes. Cependant, gouverner un réseau de maisons peut aussi servir à diminuer les inégalités entre les maisons et les personnes de la même famille et ainsi les rapprocher socialement. La construction des « maisons diasporas » accompagne les allées et venues des propriétaires entre Haïti et l'étranger. Faire une « maison diaspora » nécessite de mobiliser un réseau de personnes. C'est une manière de redistribuer « l'argent diaspora », même si cette répartition peut prendre la forme de nourriture ou de boisson.

Dans le cas des Haïtiens vivant aux États-Unis, Karen Richman montre que la maison qu'ils bâtissent à leur retour en Haïti sert à démontrer le succès de leur migration.

«Une nouvelle maison est le symbole le plus visible de la réussite des migrants à l'étranger et de leur fidélité à la patrie (Haïti). Dans le lakou où j'ai résidé, par exemple, la moitié des maisons, y compris celle que je louais, avait récemment été construite par des immigrants, dont deux ont 
pu retourner voir leur maison pendant au moins cinq ans. Trois autres « maisons de vacances » appartenant à des migrants étaient en construction, un processus qui, en l'absence de prêts hypothécaires, prend généralement des années d'investissement. Les nouvelles maisons, financées par les envois de fonds, se démarquent des maisons plus anciennes en bois, couvertes de mortier, avec toit de chaume et sol en terre battue. Ce sont des bâtiments faits de blocs de ciment, au plancher cimenté et à la toiture en tôle d'aluminium, aux structures extravagantes comme des planchers de tuiles, des toits en béton, et des balcons en fer forgé » (Richman 2005 : 74).

Comme le révèle Richman, il y a des « maisons diasporas » mises en location, au moins pour certaines chambres ou étages, à l'image de la maison dans laquelle j'ai été logé à Fonds-des-Nègres, sous la surveillance de Jérôme. La construction de cette maison a pris cinq ans. Jérôme a aussi embauché des maçons, des électriciens, des plombiers, des peintres, etc : la maison est donc aussi une source d'activité pour divers corps de métiers. Elle génère ainsi une « économie diaspora » pour ceux qui sont restés sur place, contribuant à dynamiser l'économie locale. En Haïti, les loyers sont généralement payés chaque année ou tous les six mois. Le propriétaire peut voyager une fois ou plus par an pour encaisser des paiements et profiter ses vacances dans le pays, ou bien c'est le gardien responsable de la maison qui recueille l'argent pour le déposer à la banque ou l'envoyer par virement bancaire au propriétaire vivant à l'étranger.

Les « maisons diasporas » sont cependant plus qu'un commerce. Elles sont essentielles au bien-être social de la famille. Les décisions qui président à leur construction ne sont pas le fruit d'un processus individuel. En effet, de nombreuses questions se posent : Où construire? Qui sera chargé de surveiller les travaux? D'où viennent les ressources? Et pourquoi construire ? Si la construction des «maisons diasporas » est collective, c'est qu'elle requiert des négociations avec la famille, surtout lorsque l'individu est marié et a des enfants nés à l'étranger. Ceux-ci sont parfois appelés à se prononcer, parce que généralement ils n'ont pas l'intention de revenir en Haïti, en raison de la situation précaire du pays (insécurité sociale et sanitaire, déstabilisation socioéconomique et politique, etc.).

Des « membres diasporas » de la même famille peuvent réunir des fonds pour construire une «maison diaspora », à la façon d'une entreprise familiale. Certains participent à la construction non pas en mobilisant des ressources financières, mais par exemple, en envoyant des «meubles » et des « décorations diasporas » une fois les travaux achevés. J'ai aussi connu des familles qui ont décidé de construire une « maison diaspora » parce que d'autres l'avaient fait, ce qui leur conférait du prestige.

La logique de la maison associée à la dimension familiale mobilise généralement plusieurs générations. Comme l'indique le cas de Jinette, les gens qui 
bâtissent des «maisons diasporas » ne le font pas eux-mêmes directement, parce que souvent ils ne reviennent pas en Haïti : la construction de ces maisons est donc un acte collectif. La maison révèle ainsi la configuration des relations familiales. En ce sens, elle est aussi pour ceux qui y habitent un mode de vie, un capital culturel et social. Elle est un outil à la fois économique et symbolique. Leur confort est très important pour ceux qui restent et ceux qui partent.

Les gens ne se déplacent pas seulement entre pays mais aussi entre maisons, parmi les galeries, jardins, salons, cuisines, salles de bains et chambres à coucher. Le mobilier bouge aussi : il y a une circulation constante d'aliments, de marchandises, d'objets en tout genre (appareils électroménagers et électrodomestiques, ustensiles et accessoires de cuisine) et d'argent entre individus et entre maisons. La recherche ethnographique montre que ces déplacements et ces échanges structurent le monde social haïtien (Joseph et Neiburg 2020). Ces maisons peuvent se trouver au même endroit ou dans des lieux différents de l'espace (trans)national haïtien. ${ }^{13}$ Dans leur recherche sur le quartier de Bel Air, à Port-au-Prince, en Haïti, Federico Neiburg, Natacha Nicaise et Pedro Braum montrent que:

« Les résidences sont également plus souples. Dans celles-ci, les gens ne vivent pas seulement, ils passent aussi : les femmes commerçantes qui se déplacent (entre Port-au-Prince et l'arrière-pays ou entre Port-au-Prince et les pôles commerciaux haïtiens situés à l'extérieur du pays, tels que Miami, Santo-Domingo et Panama); les hommes qui émigrent pour travailler en République dominicaine ou qui vivent aux États-Unis et sont peut-être de retour au pays » (Neiburg, Nicaise et Braum 2011 : 18).

Dès l'enfance, les gens circulent parmi les maisons jouant dans une maison, prenant leurs repas dans une autre, se baignant et dormant dans une troisième. À Fonds-des-Nègres, mes interlocuteurs distinguaient entre ceux qui fréquentaient la maison et ceux qui faisaient partie d'elle. Ces deux verbes, «fréquenter » et « faire partie de », nous permettent de comprendre la dynamique de la sociabilité et des liens affectifs dans les maisons entre les personnes qui habitent celles localisées en Haïti et à l'étranger, et aussi entre "maisons diasporas » et «maisons locales». Ceux qui «fréquentaient» les maisons s'y rendaient pour se laver, manger, boire, jouer ou parler : ce sont les habitués de la maison. Ceux qui en « faisaient partie » y avaient leurs quartiers, même s'ils étaient à l'étranger. Certains, même en diaspora, sont considérés comme «faisant partie » de la maison, car ils y ont une chambre et des biens, en plus

13 Ce mouvement à l'intérieur et entre les maisons ne se produit pas qu'en Haïti mais aussi chez les Haïtiens au Brésil et en Guyane. 
des liens affectifs forts avec des personnes qui l'habitent. «Faire partie » de la maison ne signifie donc pas nécessairement dormir dans la maison. Les expressions utilisées pour tenir compte de ces deux dimensions sont: pase nan kay la (« passer à la maison ») et viv nan kay la («vivre à la maison»). Les gens peuvent ainsi « fréquenter » et «faire partie de » plus d'une maison, de deux à dix, situées à la fois en Haïti et dans les espaces de la mobilité internationale haïtienne. Certaines personnes vont à la maison juste pour manger et dorment dans une autre maison, qui fait également partie du même réseau (trans)national. Dans le monde social haïtien, la participation à une configuration de maisons peut donc prendre différentes formes. On peut participer en s'engageant dans des activités quotidiennes, comme manger, dormir ou se laver, mais aussi à distance, par le truchement d'une «présence proxy »(Dalakoglou 2010), d'une présence par procuration. Construire une "maison diaspora », avoir une chambre chez un membre de la famille, laisser une partie de ses affaires en Haïti, envoyer des biens et de l'argent à ses proches : autant de moyens qui permettent de participer à distance à une configuration de maisons, d'être présent (pour les gens) tout en étant absent (physiquement).

Il faut souligner que dans le monde social haïtien, il y a des maisons dans lesquelles on ne cuisine pas tous les jours, du fait que parfois il n'y a rien à cuisiner (riz, pois, huile, viande, poisson, etc.). La nourriture joue donc un rôle crucial dans la circulation des gens. Si dans une maison il n'y a pas à manger faute d'aliments ou d'argent pour en acheter, les gens mobilisent d'autres maisons. La fréquentation d'une maison peut donc se restreindre à la cuisine. Certains vont à la cuisine qui se trouve à quelques mètres derrière ou à côté de la maison principale, y prennent leur repas et repartent, sans avoir accès à l'intérieur de la maison principale. En ce sens, « la nourriture est, par conséquent, un outil utile pour étudier la signification de la maison» (Petridou 2001 : 89). La participation à une configuration de maisons varie donc en fonction des ressources et des besoins de chacun, ce qui confère à leur organisation une réelle souplesse et leur permet d'agir comme outils de redistribution. À cet égard, on peut se demander si, dans le monde social haïtien, les configurations de maisons ne jouent pas un rôle similaire à celui des familles en Europe du Sud (Maloutas 1995 ; Allen 2006 ; Pfirsch 2011 ) : en l'absence d'un Etat-Providence fort, elles fournissent des biens et services aux populations qui en ressentent le besoin, sans toutefois remettre en cause les hiérarchies existantes.

La maison locale de Jinette et la maison diaspora de sa sœur Altamère à Fonds-des-Nègres font partie du même lakou. Dans certains cas, des maisons construites dans un lakou différent sont interconnectées les unes aux autres, faisant partie du même réseau transnational. Les maisons à l'étranger comme celle d'Altamère à Paris et Arnette à Cayenne ne sont pas appelées kay dyaspora ( «maisons diasporas »), mais kay nan diaspora («maisons en diaspora ») et kay lòt bò dlo («maisons d'outre-mer») ou kay aletranje («maisons à l'étranger»). 
Les échanges entre maisons ont lieu au sein d'une structure hiérarchique et, en même temps, autonome. Les relations entre maisons d'un même réseau ne se déploient pas de la même manière et sur la même échelle. Le degré d'intimité, le fait d'avoir ou pas un revenu régulier, les lieux (grand pays ou petit pays) de résidence sont autant de facteurs qui interviennent dans le choix de ce qui est échangé. Par exemple, Altamère, qui habite à Paris, envoyait chez sa sœur Jinette en Haïti des équipements électroménagers, des vêtements, de la nourriture et de l'argent. Altamère n'envoyait pas les mêmes choses chez sa sœur Arnette vivant à Cayenne : plutôt des produits cosmétiques et des marchandises qu'Arnette ne trouvait pas sur place ou qui lui coûtaient très cher. Jinette, à son tour, expédiait des produits auxquels ses sœurs n'avaient pas accès en Guyane ou dans l'hexagone : cremas (liqueur haïtienne), djondjon (champignons) et luil maskreti (une huile traditionnelle en Haïti pour se coiffer les cheveux, mais également utilisée à d'autres finalités). Altamère et Arnette transféraient de l'argent à Jinette parce que cette dernière n'avait pas de revenu régulier en Haïti. Jinette se servait de l'expression « nous sommes un » pour expliquer cette solidarité dans la circulation des biens et de l'argent, très particulière aux groupes de maisons qui composent les réseaux transnationaux et diasporas. Elle a également dit : «Celui qui a plus aide les autres ». « Aide » est un terme clé dans les réseaux de solidarité et d'échange. Cette solidarité consiste en ce que chaque membre du groupe contribue au fonctionnement de l'ensemble, à travers un don spontané. Pour certains de mes interlocuteurs, plus qu'une obligation, ces relations d'échanges sont considérées comme un devoir - comme on dit en kreyòl, se devwa $m$ (c'est mon devoir), mais tous ne pensaient pas pareil. Cependant, de la méfiance, parfois même des conflits traversent les maisons, surtout quand ceux restés sur place détournent l'argent qu'ils ont reçu de l'étranger.

\section{CONCLUSION}

Cet article montre comment la différenciation entre maisons éclaire le monde social haïtien, et, réciproquement, comment la circulation de la diaspora met en lumière la différenciation des maisons, les rapports entre maisons et objets, entre les maisons et les personnes qui partent et celles qui restent sur place. Cela nous permet de discuter le gouvernement de la maison dans cet univers en mobilité, les relations stratégiques entre les personnes, entre celles qui appartiennent à un même réseau de maisons, ainsi que les hiérarchies entre maisons.

Les récits des habitants, en particulier ceux de Jinette et de Fanfan, font envisager la maison comme un lieu symbolique et matériel, lieu habité et vécu. Si pour certains la « maison diaspora » est une réponse à la précarité de logement connue avant leur départ à l'étranger, pour d'autres, elle montre le succès 
de la migration. La maison est le lieu idéal pour maintenir des liens affectifs et familiaux, et avec Haïti, même à distance. En même temps, elle symbolise la transformation du statut socioéconomique, résultant de la mobilité. Les maisons apparaissent comme inséparables des trajectoires migratoires. Par conséquent, dans cette recherche, la maison n'est pas perçue comme un espace physique, comme un lieu indépendant. Je suis d'accord avec Elia Petridou quand elle dit « dans une ère de mobilité croissante, étudier la maison dans un espace défini devient encore plus problématique, car elle limite son utilisation comme outil d'analyse et fournit peu de poids conceptuel » (Petridou 2001 : 88). En ce sens, dans le cas des personnes étudiées, la maison apparaît comme un élément de (dis)continuité de la diaspora. Il n'y a pas de diaspora sans la mobilité des personnes et il n'y a pas de mobilité sans les maisons.

J'ai essayé de décrire et d'analyser à un double niveau la construction des maisons en Haïti et les relations entre celles-ci et celles de la diaspora. Lapproche défendue dans ce travail dé-essentialise la maison (Guedes 2017), évite de considérer la maison comme quelque chose de prêt, fini et fixé ou comme une unité repliée sur elle-même. Les maisons, en particulier les «maisons diasporas », ne sont jamais finies, les personnes diasporas cherchent toujours à aménager de nouveaux espaces, de nouveaux objets, contribuant ainsi à la valeur morale, culturelle, sociale et économique de la maison.

Ainsi, l'apparence d'une maison, son état physique, ses meubles et ses appareils électroménagers indiquent si une maison est ou non dyaspora. Mais en Haït, les familles ont toutes des proches à l'étranger. Toutes les maisons, sans distinction, ont des liens avec la diaspora, à différentes échelles. Il est donc impossible de trouver une maison qui ne soit pas influencée par la diaspora haïtienne, ce qui nuance l'opposition frontale entre « maisons diasporas » et «maisons locales».

Ainsi, c'est dans la maison qu'on prend la décision de migrer, c'est vers elle que l'on transfère de l'argent et c'est à partir d'elle que les objets en provenance de l'étranger sont redistribués. La maison est l'espace de la mobilité par excellence, en exprimant la diversité des trajectoires individuelles, collectives et sociales des personnes et des objets. La maison est un lieu de pouvoir : pouvoir manger, pouvoir dormir, pouvoir être en mobilité. Elle est aussi un lieu de savoir, savoir-circuler, savoir-faire, savoir-être-diaspora. Faire partie d'un réseau de maisons peut être une étape dans le processus de la mobilité pour devenir un jour une « personne diaspora ». 


\section{BIBLIOGRAPHIE}

ALLEN, Judith, 2006, "Welfare regimes, welfare systems and housing in southern Europe", European Journal of Housing Policy, 6 (3) : 251-277.

ARAOS, Consuelo, 2016, "When family lives nearby : kinship, socioeconomic conditions and residential configurations in Santiago, Chile", Cuadernos ISUC, 1 (1) : 1-18.

BARTHÉlemY, Gérard, 1990, L'Univers Rural Haïtien : Le Pays en Dehors. Paris : Editions l'Harmattan.

CLIFFORD, James, 1994, "Diasporas", Cultural Anthropology, Further Inflections : Toward Ethnographies of the Future, 9 (3) : 302-338.

COHEN, Robin, 1987, The New Helots : Migrants in the International Division of Labour. Aldershot: Gower.

COHEN, Robin, 1997, Global Diasporas : An Introduction. Londres, UCL Press.

CORTADO, Thomas Jacques, 2019, "Meia-água : producing space and kinship in an irregular housing subdivision in Rio de Janeiro", Artículo - Journal of Urban Research, 20 : 1-21. CORTADO, Thomas Jacques, 2020, « Maison», Anthropen.org. Paris, Editions des Archives Contemporaines, < https://www.anthropen.org/voir/Maison > (dernière consultation en octobre 2020).

DALAKOGLOU, Dimitris, 2010, "Migrating-remitting-'building'-dwelling : house-making as 'proxy' presence in postsocialist Albania", Jornal ofthe Royal Anthropological Institute, $16(4): 761-777$

FOUCAULT, Michel, 1984, «L'éthique de soi comme pratique de liberté », Dits et Ecrits, IV : 728-729.

GILROY, Paul, 1993, The Black Atlantic : Modernity and Double Consciousness. Londres : Verso. GUEDES, André Dumans, 2018, "Des-substancializando a casa", Parte II : Configurações, Prolongamentos, Mobilidades e Descontroles (dans la presse).

HALL, Stuart, 1993, "Cultural identity and diaspora", dans Patricia J. Williams et Laura Chrisman (dirs.), Colonial Discourse and Post-colonial Theory : A Reader. Londres: Harvester Wheatsheaf, 222-237.

JOSEPH, Handerson, 2015a, Diáspora : As Dinâmicas da Mobilidade Haitiana no Brasil, no Suriname e na Guiana Francesa. Rio de Janeiro : PPGAS, Museu Nacional/Universidade Federal do Rio de Janeiro, thèse de doctorat en Anthropologie Sociale, < https:/www.aca demia.edu/15267521/Tese_de_doutorado_Diaspora._As_din\%C3\%A2micas_da_mobili dade_haitiana_no_Brasil_no_Suriname_e_na_Guiana_Francesa_?auto=download $>$ (dernière consultation en octobre 2020).

JOSEPH, Handerson, 2015b, "Diáspora : sentidos sociais e mobilidades haitianas", Horizontes Antropológicos, Porto Alegre, 21 (43) : 51-78, < https://www.scielo.br/pdf/ha/ v2 ln43/0104-7 183-ha-21-43-0051.pdf > (dernière consultation en octobre 2020).

JOSEPH, Handerson, 2017a, «Diaspora, circulation et mobilité : les jeunes haïtiens au Brésil », dans Louis HernsMarcelin, Toni Cela et Henri Dorvil(dirs.), Les Jeunes Haïtiens dans les Amériques // HaitianYouth in the Americas. Montréal, Presses de l'Université du Québec-PUQ, 173-204.

JOSEPH, Handerson, 2017b, "Diáspora, refugiado e migrante : perspectiva etnográfica em mobilidade e transfronteiriça", Sociedade e Cultura, 20 (2) : 173-192, < https://www.revistas.ufg.br/fchf/issue/view/1980/showToc > (dernière consultation en octobre 2020).

JOSEPH, Handerson, 2019a, "Diáspora”, dans Federico Neiburg (dir.), Conversas Etnográficas Haitianas. Rio de Janeiro, Papéis Selvagens Edições, 229-249. 
JOSEPH, Handerson, 2019b, « Mobilité transfrontalière haïtienne au Brésil : kongo, vyewo et dyaspora », dans Dimitri Béchacq, Fritz Calixte, et Marie Meudec (dirs.), Les Migrations et la Caraïbe : (Dés)ancrages, Mouvements et Contraintes. Paris, L'Harmattan, 8 : 207-231.

JOSEPH, Handerson, 2020, "The haitian migratory system in the Guianas : beyond borders ” , Diálogos, 24 (2) : 198-227, < http://periodicos.uem.br/ojs/index.php/Dialogos > (dernière consultation en octobre 2020).

JOSEPH, Handerson, et Federico NEIBURG, 2020, " I'm going to die in the street : Haitian lives in the pandemic ", City \& Society, < https://anthrosource.onlinelibrary.wiley.com/ doi/epdf/10.1111/ciso.12314 > (dernière consultation en octobre 2020).

LÉOBAL, Clémence, 2019, "The making of urban maroons", Artículo - Journal of Urban Research, $20: 1-21$.

L'ESTOILE, Benoît de, 2014, "Money is good, but a friend is better: uncertainty, orientation to the future, and 'the economy'", Current Anthropology, 55 (9) : 62-73.

MALOUTAS, Thomas, 1995, «Ségrégation urbaine et relations familiales dans deux villes grecques : Athènes et Volos », Sociétés Contemporaines, 22/23 : 89-106.

MARCELIN, Louis Herns, 1996, L'Invention de la Famille Afro-Américaine : Famille, Parenté et Domesticité Parmi les Noirs du Recôncavo da Bahia, Brésil. Rio de Janeiro : PPGAS, Museu Nacional/ Universidade Federal do Rio de Janeiro, thèse de doctorat en Anthropologie Sociale.

MARCELIN, 1999, "A linguagem da casa entre os negros no Recôncavo da Bahia", Mana, $5(2): 31-60$.

MÉTRAUX, Alfred, 1958, Le Vaudou Haïtien. Paris : Gallimard.

MORAL, Paul, 1961 [1978], Le Paysan Haïtien : Etude sur la Vie Rurale en Haïti. Port-au-Prince : Editions Fardins.

MOTTA, Eugênia, 2014, "Houses and economy in the favela", Vibrant - Virtual Brazilian Anthropology, 11 (1) : 118-158.

NEIBURG, Federico, Natacha NICAISE, et Pedro BRAUM, 201 1, Lideranças em BelAir. Rio de Janeiro : NUCEC, Museu Nacional-UFRJ, rapport de recherche en Anthropologie Sociale. PERALDI, Michel, 1999, « Marseille : réseaux migrants transfrontaliers, place marchande et économie de bazar », Cultures et Conflits : Les Anonymes de la Mondialisation, 33-34 : 1-13. PERTIERRA, Anna Cristina, 2010, "Materialidade da escassez : as residências urbanas de Cuba e sua cultura material”, dans Olívia Maria Gomes da Cunha (dir.), Outras Ilhas : Espaços, Temporalidades e Transformações em Cuba. Rio de Janeiro, Aeroplano, 395-423.

PETRIDOU, Elia, 2001, "The taste of home", dans Daniel Miller (dir.), Home Possessions : Material Culture Behind Closed Doors. Oxford et New York, Berg, 87-104.

PFIRSCH, Thomas, 2011, «La localisation résidentielle des classes supérieures dans une ville d'Europe du Sud », L'Espace Géographique, 40 (4) : 305-318.

PINA-CABRAL, João de, 2014, "Agnatas, vizinhos e amigos : variantes da vicinalidade em África, Europa e América”, Revista de Antropologia, 57 (2) : 23-46.

RICHMAN, Karen, 2005, Migration and Vodou. Gainesville, FL : University Press of Florida. SAFRAN, William, 1991, "Diasporas in modern societies : myths of homeland and return". Diaspora, 1 (1) : 83-99.

SHEFFER, Gabriel, 1986, Modern Diasporas in International Politics. New York, Saint Martin Press. 